\title{
Visibility Functions of Solar and Stellar Irradiance Variations
}

\author{
Jürgen Staude and Günter Bartling \\ Astrophysikalisches Institut Potsdam, Sonnenobservatorium \\ 'Einstein-Turm', Telegrafenberg, D-14473 Potsdam, Germany
}

Yuzef D. Zhugzhda and Namig S. Dzhalilov

IZMIR RAN, Troitsk, Moscow Region, 142092 Russia

\begin{abstract}
We calculated visibility functions of intensity fluctuations due to adiabatic waves in a realistic model of a non-grey, stratified atmosphere in LTE. The results for different optical wavelengths $\lambda$ and their angular dependence are discussed; moreover, a method for selecting various low-degree $p$-modes from observations of spectral irradiance fluctuations at different $\lambda$ including the UV is suggested.
\end{abstract}

\section{Introduction}

The presence of the $p$ modes of global solar oscillations has been proved in the time series not only of velocity, but also of spectral and total irradiance observations. There are hints of a non-adiabatic behaviour as well as of the influence of the gravitational stratification.

In some recent papers we investigated non-adiabatic hydrodynamic waves in a radiative, thermally conducting, homogeneous model atmosphere in LTE for the grey case (Dzhalilov et al. 1992, 1994) and for the non-grey case as well (Zhugzhda et al. 1993). Our theoretical approach took into account the nonadiabatic effects in great universality, but future investigations should consider the basic effect of the gravitational stratification as well. In the present paper we take first steps in this direction.

\section{Intensity fluctuations due to waves in a non-grey stratified atmo- sphere}

In order to obtain the visibility of luminosity fluctuations in a non-grey, stratified atmosphere, we computed the relative intensity fluctuations $I^{\prime} / I_{0}$ for different $\lambda$ (or frequencies $\nu$ ) and positions $\mu$ on the disk. The intensity fluctuation depends on the relative fluctuations of temperature $T^{\prime} / T_{0}$ and density $\rho^{\prime} / \rho_{0}$, multiplied by the logarithmic derivatives of opacity with respect to $T$ and to $\rho$, respectively, describing the action of the opacity fluctuations $\left(f_{\chi}\right)$, and of the gradient of the source function with respect to $T$ (influence of $T^{\prime}$ on the source function, $\left.f_{S}\right)$. As a first step we consider the degree $l=0$ only and normalise 
the relative fluctuation of $I_{\nu}$ to those of $T$, the latter assumed constant with depth. The absolute value of that expression will be called spectral darkening function (or visibility function for $l=0): D_{\lambda}=\left|\left(I_{\nu}^{\prime} / I_{0 \nu}\right) /\left(T^{\prime} / T_{0}\right)\right|=\left|f_{S}+f_{\chi}\right|$. After integrating $I_{\nu}^{\prime}$ and $I_{0 \nu}$ over $\nu$ or the wavelength $\lambda$ we obtain the integrated darkening function $D$.

\section{Calculations for a solar model}

As an example we used the VAL3C model of the average solar atmosphere (Vernazza et al. 1981) in a recalculated form, taking into account the equation of state for a realistic solar plasma with 10 elements, considering molecules as well as non-LTE for $\mathrm{H}$ and all important opacity sources. The darkening and visibility functions have been computed for $\lambda$ from the UV up to the IR.

For the adiabatic case our calculations show small but important differences to the results of Toutain \& Gouttebroze (1993) who considered a pure H plasma and an opacity by $\mathrm{H}^{-}$only: we derive a negative visibility function in the disk centre, which means opposite phases between $T^{\prime}$ and $I^{\prime}$. This effect is strongest in the UV and decreases with increasing $\lambda$. At shorter $\lambda$ the visibility is focussed onto a smaller sphere around the disk centre, thus suggesting a new method of mode selection by observing the spectral irradiance fluctuations at different $\lambda$. This method is applicable not only to the Sun, but also to stars.

\section{Conclusion}

In the next step our modelling will be completed by introducing information on the normal modes from a model of non-radial acoustic oscillations in a realistic solar model, to begin with the adiabatic case. However, the final step should consider non-adiabatic conditions as well. Such an analysis which is under preparation will provide the basis for confronting our modelling with measurements of ground-based velocity data and of luminosity oscillations planned to be measured aboard the satellites CORONAS-F and SOHO and for suggesting new space-borne experiments.

Acknowledgments. The authors gratefully acknowledge support of the present work by the German Space Agency (DARA) under grant No. 50 QL 92072.

\section{References}

Dzhalilov, N.S., Zhugzhda, Y.D., \& Staude, J. 1994, A\&A, 291, 1001

Staude, J., Dzhalilov, N.S., \& Zhugzhda, Y.D. 1994, Solar Phys., 152, 227

Toutain, T., \& Gouttebroze, P. 1993, A\&A, 268, 309

Vernazza, J.E., Avrett, E. H., \& Loeser, R. 1981, ApJS, 45, 635

Zhugzhda, Y.D., Dzhalilov, N.S., \& Staude, J. 1993, A\&A, 278, L9 